\title{
RESEARCH ARTICLE \\ Postural Mechanisms to Control Body Displacements in the Performance of Lateral Gaze Shifts
}

\author{
Cédrick T. Bonnet ${ }^{1}$, Cédric Morio ${ }^{1}$, Sébastien Szaffarczyk ${ }^{1}$, Patrice R. Rougier ${ }^{2}$ \\ ${ }^{1}$ Laboratoire de Neurosciences Fonctionnelles et Pathologies, CNRS, Universités Lille, France. ${ }^{2}$ Laboratoire de Modélisation \\ des Activités Sportives, Université de Savoie, Le Bourget du Lac, France.
}

\begin{abstract}
Medialateral postural control mechanisms (bodyweight distribution and center of pressure location) have been studied in static conditions. Our objective was to determine how these mechanisms are adjusted to perform voluntary movements, in our case $80^{\circ}$ lateral gaze shifts at $0.125 \mathrm{~Hz}$ and $0.25 \mathrm{~Hz}$. In healthy, young adults, we expected body marker (neck, lower back) and center of pressure displacements to be significantly greater in gaze shift conditions than in the stationary gaze condition. To explain these changes in center of pressure displacement, the amplitude contribution of both mechanisms was expected to increase significantly. All these results were found accordingly. Unexpectedly, the active contribution of the bodyweight distribution mechanism was negatively related to body marker displacements in the gaze shift conditions ( $n s$ in stationary condition). Moreover, changes in the contribution of the mechanisms were statistically weaker in effect size than changes in body displacement. However, the participants were not unstable because they performed the visual tasks as requested. We propose that the strength of medialateral postural control mechanisms may not only be strengthened to control challenging ML stance conditions but also slightly weakened to allow the performance of adequate body motions in ongoing tasks.
\end{abstract}

Keywords: postural control mechanisms, postural coordination, visual tasks, medialateral axis

n stance, postural control mechanisms have to work continuously to restrain postural sway (Winter, 1995). In the medialateral (ML) axis, Winter, Prince, Stergiou, and Powell (1993) and Winter, Prince, Frank, Powell, and Zabjek (1996) proposed the existence of a bodyweight distribution and center of pressure (COP) location mechanisms. The first mechanism is performed by loading the bodyweight under one foot and thus unloading the bodyweight under the other foot. The control may be performed at the hip level (e.g., Winter et al., 1996; Winter et al., 1993). The second mechanism is performed by inversion or eversion at the ankle level. The two mechanisms were shown to play significant and complementary roles in controlling ML COP displacement (Bonnet, Mercier, \& Szaffarczyk, 2013; Gatev, Thomas, Kepple, \& Hallett, 1999; Lafond, Corriveau, \& Prince, 2004; Rougier, 2007, 2008; Termoz et al., 2008; Winter et al., 1996; Winter et al., 1993). The bodyweight distribution mechanism (referred to as loading/unloading contribution to vertical forces) was shown to be the primary mechanism when the feet are side by side. The COP location mechanism (referred to as left and right COP change) was shown to have a secondary role in explaining the ML COP displacement, sometimes significant (Bonnet et al., 2013; Termoz et al., 2008) but sometimes not significant (Winter et al., 1996; Winter et al., 1993). Former studies showed that the closer the feet are, the higher the active contribution (represented by higher cross-correlations in narrow stance than in standard stance; cf. Method) of both mechanisms is (cf. Bonnet et al., 2013; Gatev et al., 1999) with greater changes for the COP location mechanism (Bonnet et al., 2013). These results have been found in static conditions (in changing the foot position) with no focal motions to be performed. However, in upright stance, individuals are rarely static; they move and perform tasks all the time (Riccio $\&$ Stoffregen, 1988). Hence, these published studies may provide an initial but incomplete understanding of these mechanisms. We decided to investigate how the postural control mechanisms work in conditions requiring body motions.

A recent study by Bonnet, Cherraf, and Do (2014a) explained that Winter et al.'s $(1993,1996)$ model cannot be used all the time. This study showed that the anteroposterior (AP) bodyweight distribution and COP location mechanisms cannot be studied with the feet side by side. It also showed that the two mechanisms should not be studied in Tandem Romberg (with one foot forward the other). We preferred analyzing both mechanisms in the ML axis for three reasons. First, our goal was to provide representative data of everyday life and the feet side-by-side condition is more common than the Tandem Romberg condition. Second, the literature reports showed a closed link between ML postural instability and ML fall (Maki, Holliday, \& Topper, 1994) and between ML fall and hip fractures (Rogers \& Mille, 2003). Investigations to better understand the functioning of ML postural control mechanisms are thus needed. Third, this present study served to provide preliminary data to understand Parkinson's disease-related deficiencies in ML postural control.

Some studies involving ML active conditions have been published in the literature. The participants had to perform small or large ML gaze shifts to reach a single target per trial (Anastopoulos, Ziavra, Hollands, \& Bronstein, 2009; Hollands, Ziavra, \& Bronstein, 2004; Sklavos, Anastasopoulos, \& Bronstein, 2010). Other participants also had to reach a target appearing left and right at different frequencies and amplitudes (Stoffregen, Bardy, Bonnet, Hove, \& Oullier, 2007; Stoffregen,

Correspondence address: Cédrick T. Bonnet, Laboratoire de Neurosciences Fonctionnelles et Pathologies, CNRS, Universités Lille, Lille, France.e-mail: cedrick.bonnet@chru-lille.fr

Color versions of one or more of the figures in the article can be found online at www.tandfonline.com/vjmb. 
Bardy, Bonnet, \& Pagulayan, 2006). In all these studies, the authors analyzed COP displacement, postural displacement, and/or postural coordination. They could not analyze the contribution of the ML bodyweight distribution and COP location mechanisms because they only used a single force platform (for the need of two platforms; cf. Winter et al., 1993). Recently, Bonnet and Despretz (2012) used two force platforms in an active study. Twelve young adults performed two gaze shift conditions in which they had to track the appearance of a dot on the right and left at $150^{\circ}$ of visual angle and at a frequency of 0.5 or $1 \mathrm{~Hz}$. In this study, COP displacement in standard stance (feet in spontaneously chosen positions) and wide stance (feet about $40 \mathrm{~cm}$ apart) were significantly greater than in the stationary gaze condition. The two ML postural control mechanisms were not analyzed yet. In Bonnet and Despretz, the visual tasks engaged a great amount of behavioral variability. We decided to investigate easier visual tasks that could be performed later by patients with Parkinson's disease and their healthy older controls.

In the present study, we sought to determine how the ML bodyweight distribution and COP location mechanisms were adjusted to perform $80^{\circ}$ lateral gaze shifts at $0.125 \mathrm{~Hz}$ and $0.25 \mathrm{~Hz}$. We expected ML body displacements (lower back, neck) and COP displacement to be significantly greater in gaze shift conditions as a result of body (head notably) motions to perform the task (Hypothesis 1). For the control of ML stance, the contribution of the ML bodyweight distribution and COP location mechanisms was expected to be greater (Hypothesis 2) and more significantly related to ML body displacements in the gaze shift conditions than in the stationary gaze condition (Hypothesis 3 ). In the nonchallenging stationary gaze condition, the mechanisms were not expected to be significantly related to body displacement since the mechanisms are robust to changes (Termoz et al., 2008). More significant findings were expected in the $0.25 \mathrm{~Hz}$ condition than in the $0.125 \mathrm{~Hz}$ condition (Hypothesis 4), as the former condition required twice more gaze shifts and supposedly greater postural control adjustments than the latter one.

\section{Method \\ Participants}

Sixteen healthy, students from the Universities of Lille participated in this study. They were included because none of them had a history of neurological or musculoskeletal disease, vestibular problems or recurrent dizziness. All gave their written, informed consent to participation and the study was performed in accordance with the tenets of the Declaration of Helsinki. The group's mean age, bodyweight and height were $21.59 \pm 2.65$ years, $61.94 \pm$ $10.08 \mathrm{~kg}$, and $1.72 \pm 0.10 \mathrm{~m} \mathrm{~s}$, respectively.

\section{Apparatus}

A dual-top force platform (AMTI, Watertown, MA) was used to record forces and moments under each foot. The platform was placed $1.50 \mathrm{~m}$ from the center of a panoramic display $\left(180^{\circ}\right.$, radius: $2.1 \mathrm{~m}$; height: $2.1 \mathrm{~m}$; Figure 1$)$. Its sampling frequency was set to $120 \mathrm{~Hz}$. Printed lines marked the position of the heels and big toes (the subjects were barefoot) to get a stance width of $14 \mathrm{~cm}$ and stance angle of $17^{\circ}$ (normative values by McIlroy \& Maki, 1997).

A two-camera motion analysis system (Version 7.5, SIMI Reality Motion Systems GmbH, Munich, Germany) was used to record body marker displacements (marker diameter: $2.5 \mathrm{~cm}$ ) with a sampling frequency of $15 \mathrm{~Hz}$. The reflective markers were attached to the back of a hip belt (the lower back marker), of the neck (the neck marker), and of a headset (the head marker). Special lights on each of the two cameras (Led Lenser P3 8403, LED torch) were used to light the markers.

A head-mounted eye tracker (SensoMotoric Instruments, Teltow, Germany) was attached to the headset worn by the participants (Figure 2). On the computer screen, the video showed the visual environment and (as a cross) what the right eye was looking. The iViewX system recorded the pupil position at a sampling rate of $50 \mathrm{~Hz}$.

A computer software projected a black dot $\left(5^{\circ}\right.$ of visual angle) onto the panoramic display (Figure 1) at the participant's eye height.

All apparatus were synchronized.

\section{Conditions}

In the stationary control gaze condition, the participants had to look at the stationary dot in front of them (Figure 1).

\footnotetext{
drawn to scale). The participant stood on a dual-top force platform in the experimental room at a distance of $1.50 \mathrm{~m}$ from a semicircular, panoramic display. The target (a black dot, subtending a visual angle of $5^{\circ}$ ) was presented either in a stationary position in front of the participant or alternately on the left and right at a visual angle of $80^{\circ}$. The dot's position was recorded with a computer program and projected onto the screen by three cameras.
}

FIGURE 1. Description of the experimental setting (not 


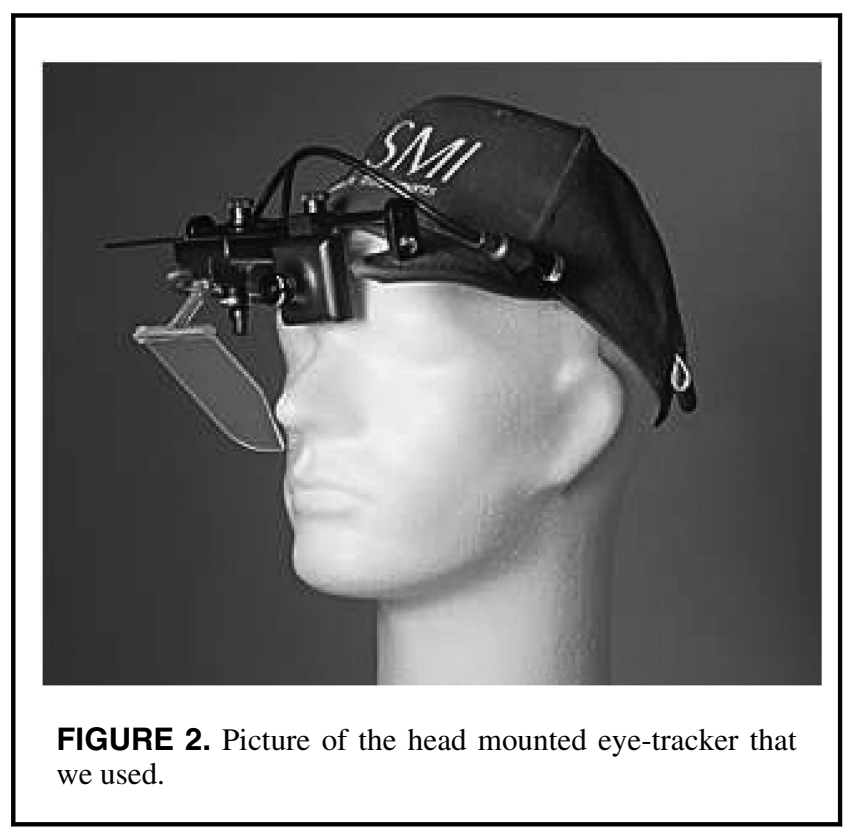

In the two gaze shift conditions, the participants had to track a dot that appeared alternately to their left and right at a visual angle of $80^{\circ}$ (Figure 1) at $0.125 \mathrm{~Hz}$ or $0.25 \mathrm{~Hz}$. We decided to perform two visual tasks and not only one to test whether ML postural control could be different in a very slow visual task (the $0.125 \mathrm{~Hz}$ condition) and in a slow visual task (the $0.25 \mathrm{~Hz}$ condition). These data will also be relevant for our future studies with older adults and patients with Parkinson's disease.

In the $0.125 \mathrm{~Hz}$ and $0.25 \mathrm{~Hz}$ conditions, gaze shifts had to be performed quickly once the target had disappeared but with no anticipation (cf. Bonnet \& Despretz, 2012). In each condition, the participants had to relax with their arms extended along their side. During trials, the participants were aware that they had to continuously look through the small window of the eye tracker (cf. Figure 2) to perform each single gaze shift; no eye data could be recorded otherwise. Thus, they understood that their head had to rotate their gaze quickly about $80^{\circ}$ to reach the target in the way they found it most comfortable (that is with their head or trunk or combined motions, instruction not suggested to the participants yet). There were four trials (32 s each) per visual condition to ensure adequate statistical power. The order of the conditions was randomized.

\section{Dependent Variables}

COP and body marker displacements. As in former works, the standard deviation (SD), range, and mean velocity were used to analyze COP and body marker displacements (e.g., Bonnet \& Despretz, 2012; Day, Steiger, Thompson, \& Marsden, 1993; Mouzat, Dabonneville, \& Bertrand, 2004). For control purposes, the COP and body marker positions as well as the bodyweight distribution were analyzed (cf. Rougier, 2007; Tarantola, Nardone, Tacclini, \& Schieppati, 1997).

Contribution of the ML bodyweight and COP location mechanisms. With one single force platform, it is not possible to measure the loading/unloading of bodyweight (referred to as $\mathrm{COP}_{v}$ in the model computation) and the COP displacement under each foot (reffered to as $\mathrm{COP}_{c}$, cf. Winter et al., 1993). For investigating the two mechanisms, we used our dual-top force platform and an updated version (Rougier, 2007, 2008) of the validated model of ML postural control (Lafond et al., 2004; Termoz et al., 2008; Winter et al., 1993, 1996):

$$
\begin{aligned}
& C O P_{n e t}(t)=\operatorname{COP}_{l}(t) \frac{R_{v l}(t)}{R_{v l}(t)+R_{v r}(t)} \\
& +\operatorname{COP}_{r}(t) \frac{R_{v r}(t)}{R_{v l}(t)+R_{v r}(t)} \\
& \operatorname{COP}_{c}(t)=C O P_{l}(t) \times \text { mean }_{v l}+\operatorname{COP}_{r}(t) \times \text { mean }_{v r} \\
& \operatorname{COP}_{v}(t)=\operatorname{meanCOP}_{l} \frac{R_{v l}(t)}{R_{v l}(t)+R_{v r}(t)} \\
& + \text { meanCOPr } \frac{R_{v r}(t)}{R_{v l}(t)+R_{v r}(t)}
\end{aligned}
$$

In the three equations, $\mathrm{COP}_{l}(\mathrm{t})$ and $\mathrm{COP}_{r}(\mathrm{t})$ are the $\mathrm{COP}$ displacement under the left and right foot, respectively. $\mathrm{COP}_{\text {net }}$ is the resultant COP displacement under both feet. $\mathrm{R}_{v l}(\mathrm{t})$ and $\mathrm{R}_{v r}(\mathrm{t})$ are the vertical reaction forces under the left and right foot, respectively. MeanCOP $\mathrm{M}_{l}$ meanCOP $r$, meanR $_{v l}$, and meanR $\mathrm{R}_{v r}$ are the mean of each time series.

Equation 1 simply shows how to compute the COP displacement with two force platforms (cf. Winter et al., 1996). In Equation 2, the $\mathrm{COP}_{c}$ displacement was calculated by eliminating the $\mathrm{COP}_{\text {net }}$ displacement explained by the $\mathrm{COP}_{v}$ displacement (constant mean of bodyweight under both feet throughout the trial). In Equation 3 the $\mathrm{COP}_{v}$ displacement was calculated by eliminating the $\mathrm{COP}_{\text {net }}$ displacement explained by the $\mathrm{COP}_{c}$ displacement (constant mean of COP location under both feet throughout the trial). Figure 3 shows the result of these equations for one trial, as an example. This mathematical model is explained in former published articles (e.g., Rougier, 2007, 2008; Winter et al., 1996).

The three time series from the above mathematical model are the $\mathrm{COP}_{\text {net }}$ (Equation 1), the $\mathrm{COP}_{c}$ (Equation 2) and the $\mathrm{COP}_{v}$ time series (Equation 3). These $\mathrm{COP}_{c}$ and $\mathrm{COP}_{v}$ time series then need to be analyzed to know the contribution of each mechanism to explain the COP displacement $\left(\mathrm{COP}_{\text {net }}(\mathrm{t})\right)$. To do so, two complementary analyses were performed.

The first analysis was concerned with the amplitude contribution, or strength, of the mechanisms. We analyzed the absolute amplitude contribution represented by the variability of $\mathrm{COP}_{c}(\mathrm{t})$ and $\mathrm{COP}_{v}(\mathrm{t})$ with no reference to the variability of $\mathrm{COP}_{\text {net }}(\mathrm{t})$. In our study, this analysis was useful to investigate changes in the amplitude contribution of the 


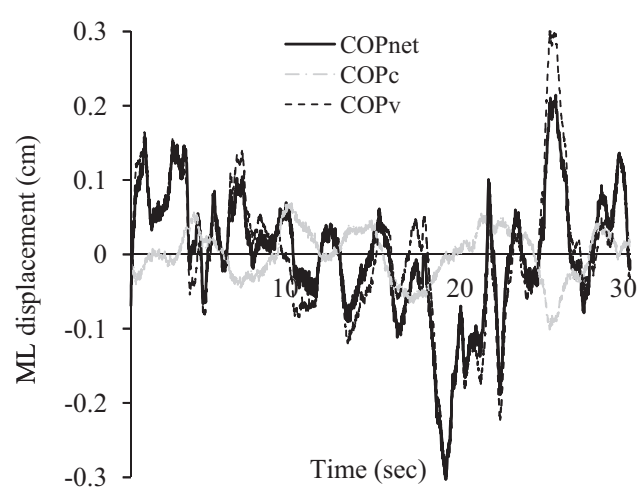

FIGURE 3. Time series for COPnet, $\mathrm{COP} v$, and $\mathrm{COP} c$ in one trial (30 s long) performed by the experimenter (units: $\mathrm{cm}$ ) in the medialateral (ML) axis. The COPnet is the integrated displacement of the COP under both feet. The COPv (t) is the component of the COPnet(t) that can be explained by the bodyweight mechanism. The $\mathrm{COP} c(\mathrm{t})$ is the component of the COPnet(t) that can be explained by the center of pressure location mechanism.

mechanisms from the static to the active conditions. We performed this analysis with $\mathrm{SD}\left(\mathrm{SD} \mathrm{COP}_{c}(\mathrm{t})\right.$ and $\mathrm{SD}$ $\left.\mathrm{COP}_{v}(\mathrm{t})\right)$ as in Rougier $(2007,2008)$. We also compared the $\mathrm{SD}$ of $\mathrm{COP}_{c}$ and $\mathrm{COP}_{\text {net }}$ time series and of $\mathrm{COP}_{v}$ and COPnet time series. This analysis was called relative amplitude contribution because it is relative to $\mathrm{COP}_{\text {net }}(\mathrm{t})$. The two variables representative of this relative amplitude contribution were $\% S D C O P_{v} / C O P_{\text {net }}$ and $\% S D C O P_{v} / C O P_{\text {net. }}$. This analysis was useful to investigate changes in the amplitude contribution of the mechanisms relative to the COP displacement to be explained.

The second analysis calculated the cross-correlation coefficient between $\mathrm{COP}_{c}(\mathrm{t})$ and $\mathrm{COP}_{v}(\mathrm{t})$ on the one hand and $\mathrm{COP}_{\text {net }}(\mathrm{t})$ on the other hand (denoted as $\mathrm{COP}_{c}$ vs. $\mathrm{COP}_{\text {net }}$ and $\mathrm{COP}_{v}$ vs. $\mathrm{COP}_{\text {net }}$, respectively; Gatev et al., 1999; Lafond et al., 2004; Rougier, 2007, 2008; Termoz et al., 2008; Winter et al., 1996; Winter et al., 1993). This analysis compared the similarity of the $\mathrm{COP}_{c}$ versus $\mathrm{COP}_{\text {net }}$ and $\mathrm{COP}_{v}$ versus $\mathrm{COP}_{\text {net }}$ time series, both in terms of direction and proportionality of the time series. These analyses were not concerned with the respective amplitudes of the time-series (as in the first analysis; cf. Bonnet, Cherraf, Szaffarczyk \& Rougier, 2014b). As proposed by Bonnet et al. (2014a; 2014b), these cross-correlation analyses may be concerned with the degree of active contribution of the mechanism to control the $\mathrm{COP}_{\text {net }}$ displacement - whether the mechanisms were sufficiently active or not. As proposed by Bonnet et al. (2014a, 2014b), we assumed that the higher the cross-correlation coefficient, the higher the active contribution of the postural mechanism to controlling the ML COP displacement.

For the analyses, both changes in the amplitude and active contributions were relevant to discuss the overall contribution of that mechanism. This overall contribution was calculated in taking into account both the amplitude and active contributions of each mechanism. If both amplitude and active contributions were higher or lower in one condition than in another condition, the overall contribution was assumed to be higher or lower, respectively. If both amplitude and active contribution had opposite trends (one getting higher, the other getting lower), we compared the effect sizes of each analysis to decide whether the overall contribution was higher or lower in the first condition than in the second condition. Indeed, we had no reason to assume that the amplitude contribution was more important than the active contribution, or vice versa, to discuss the overall contribution of the ML bodyweight distribution and COP location mechanisms.

Eye-in-orbit, head-on-trunk, and trunk-in-space angular displacements. The video of the eye tracker was not recorded but it could be watched online by the experimenter during trials. Hence, the experimenter could ask the participant to repeat one trial if the task was not well performed. The participants performed the task as requested if they moved the eyes from the disappearing target directly to the appearing target, thus at the expected amplitude, frequency and timing. The detail of the eye position was recorded.

The eye-in-orbit angular displacement corresponded to the angle formed by the eye relative to the orbit referential. This angle was obtained in using the ML linear displacement of the eye and the inverted tangent function. The head-in-space and trunk-in-space angular displacements corresponded to the planar yaw angles of the head-neck vector and the neck-lower back vector projected on the horizontal plane, respectively. These angular displacements were obtained from the SIMI Reality Motion System software directly. Because this system recorded data relative to the earth reference, the head-on-trunk angular displacement was obtained by subtracting the trunk-in-space angular displacement from the head-in-space angular displacement.

The time series of eye-in-orbit and head-on-trunk angular displacements (left-right turns) and stationary position (to look at the target) were clearly pictured in each single trial. An example of head-on-trunk angular displacement time series in a $0.25 \mathrm{~Hz}$ condition is shown in Figure 4. We calculated the mean angular positions of the eye-in-orbit and head-on-trunk when the target was on the right and left (between the vertical lines on Figure 4). The resulting left and right mean angles (two and four mean angles in the 0.125 and $0.25 \mathrm{~Hz}$ conditions, respectively) were averaged for each trial. The time series of trunk-in-space angular displacement were subject-dependent. These later time series showed clear transition and stationary time series in two participants, they were related to the target-or head-motion and half-time completely random in seven participants and they were completely random in seven participants. The Figure 4 shows a completely random-not related to the target position-time series of trunk-in-space angular displacement. To calculate the mean left and right angular positions 


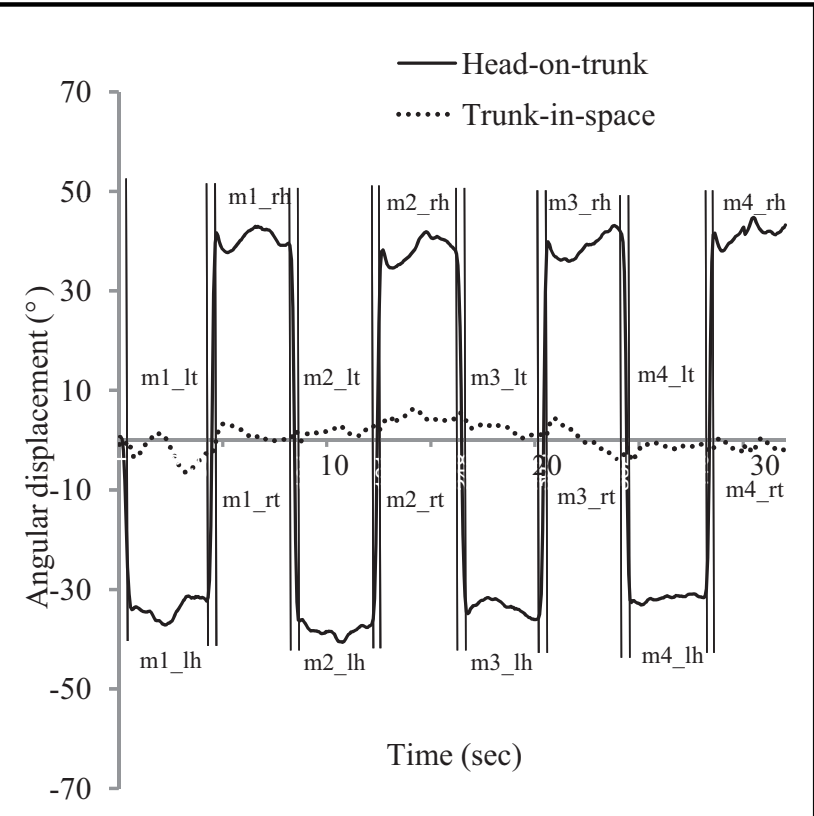

FIGURE 4. Time series for the head-on-trunk angular displacement and trunk-in-space angular displacement (units: deg $=$ degrees) in one trial (32 s long) performed by one participant in the $0.25 \mathrm{~Hz}$ condition. The left and right means position of the head-on-trunk angular displacement were calculated with the four mean angular positions when the head had finished to turn to reach the left and right targets, respectively. The eight mean angular positions of the trunk-in-space angular displacement were calculated at the same moment that the eight mean angular positions of the head-in-space angular displacement. For the abbreviations: $\mathrm{m} 1 \_\mathrm{lh}=$ mean 1 when the head was on the left; $\mathrm{m} 2$ rh $=$ mean 2 when the head was on the right; m1_lt = mean 1 when the trunk was on the left; $\mathrm{m} 3$ _lt $=$ mean 3 when the trunk was on the right.

of the trunk-in-space, we looked at the two and four similar time series as for the head time series (same beginning and same end) in the $0.125 \mathrm{~Hz}$ and $0.25 \mathrm{~Hz}$ conditions, respectively. In our example in Figure 4, the four left means (m1_lt, m2_lt, m3_lt, m4_lt) and the four right means (m1_rt, m2_rt, m3_rt, m4_rt) of trunk-in-space angular displacement were calculated at the same moment as the four left means (m1_lh, m2_lh, m3_lh, m4_lh) and the four right means (m1_rh, m2_rh, m3_rh, m4_rh) of head-on-trunk angular displacement.

\section{Data Analysis}

All the dependent variables (COP displacement, body and angular displacement, amplitude and active contributions of ML bodyweight and COP location mechanisms) were analyzed in the ML axis.

All the dependent variables were normally distributed and there was no outlier. Comparisons between conditions were performed with one-way repeated measures analyses of variance (ANOVAs). Pearson's correlations were used to test linear relationships between (a) the contribution of both mechanisms and (b) the body marker displacements and angular displacements in each condition. All analyses were performed at $p<.01$.

\section{Results}

\section{Visual Performance: Gaze Shifts Well Performed}

During the experimental session, the experimenter continuously verified that the participants kept their eyes fully on the target in the stationary gaze condition and that they reached each single target in the 0.125 and $0.25 \mathrm{~Hz}$ conditions. As in Bonnet and Despretz (2012), no trial was repeated and the targets were easily reached at the requested amplitude, frequency and timing.

\section{Effects of Conditions on Eye-in-Orbit, Head-on-Trunk, and Trunk-in-Space Angular Displacements}

The eye-in-orbit angular displacement was significantly greater in the $0.125 \mathrm{~Hz}\left(10.17 \pm 5.61^{\circ}\right)$ than in the $0.25 \mathrm{~Hz}$ conditions $\left(8.55 \pm 5.59^{\circ}\right), F(1,15)=10.89, p<.05$. The eyes almost did not move in the stationary gaze condition $\left(1.35 \pm 0.52^{\circ}\right)$.

The head-on-trunk and trunk-in-space angular displacements were similar between the two gaze shift conditions, $\left.F_{s}(1,15)<1.15, p>.05\right)$. On average in both tasks, the mean head-on-trunk and trunk-in-space angular displacements were $58.44 \pm 10.58^{\circ}$ and $12.20 \pm 10.07^{\circ}$, respectively. These values were greater than the ones in the stationary gaze condition $\left(0.25 \pm 0.65^{\circ}, 0.21 \pm 0.64^{\circ}\right)$.

Overall therefore, healthy, young adults performed $80^{\circ}$ gaze shifts mostly in turning their head (almost three quarters of body angular displacement) and additionally their eyes and trunk.

\section{Effects of Conditions on COP and Body Linear Displacements and on the Contribution of the ML Bodyweight and COP Location Mechanisms}

The results of the ANOVA are reported in Table 1. Overall, the results showed that the participants exhibited significantly higher amplitude and velocity of body and COP displacement and higher absolute amplitude contribution of the ML bodyweight and COP location mechanisms in the active conditions than in the stationary gaze condition.

\section{Contributions of the Mechanisms Significantly Related to Body Displacements?}

The results of the Pearson's correlation analyses are reported in Table 2. These analyses showed that there were more significant relationships between body displacements and the contributions of the two mechanisms in the active gaze shift conditions than in the stationary gaze condition. 
TABLE 1. Results of the Repeated Measures ANOVA and Post Hoc Newman-Keuls Analyses

\begin{tabular}{lcccc}
\hline \hline & $0.25 \mathrm{~Hz}$ & $0.125 \mathrm{~Hz}$ & Stationary & ANOVA \\
\hline $\mathrm{R}$ of COP displacement $(\mathrm{cm})$ & $1.92( \pm 1.01)^{\mathrm{a}}$ & $1.84( \pm 0.93)^{\mathrm{a}}$ & $1.10( \pm 0.48)$ & $F(2,30)=11.80, n_{p}^{2}=.31, p<.01$ \\
SD of COP displacement $(\mathrm{cm})$ & $0.37( \pm 0.22)^{\mathrm{a}}$ & $0.40( \pm 0.25)^{\mathrm{a}}$ & $0.22( \pm 0.11)$ & $F(2,30)=8.47, n_{p}^{2}=.27, p<.01$ \\
V of COP displacement $\left(\mathrm{cm} . \mathrm{s}^{-1}\right)$ & $1.80( \pm 0.23)^{\mathrm{a}}$ & $1.74( \pm 0.25)^{\mathrm{a}}$ & $0.66( \pm 0.26)$ & $F(2,30)=12.88, n_{p}^{2}=.32, p<.01$ \\
$\mathrm{R}$ of neck displacement $(\mathrm{cm})$ & $4.32( \pm 1.52)^{\mathrm{a}}$ & $4.40( \pm 1.87)^{\mathrm{a}}$ & $1.30( \pm 0.57)$ & $F(2,30)=49.10, n_{p}^{2}=.43, p<.01$ \\
SD of neck displacement $(\mathrm{cm})$ & $1.21( \pm 0.44)^{\mathrm{a}}$ & $1.27( \pm 0.56)^{\mathrm{a}}$ & $0.31( \pm 0.15)$ & $F(2,30)=52.24, n_{p}^{2}=.44, p<.01$ \\
V of neck displacement $\left(\mathrm{cm} . \mathrm{s}^{-1}\right)$ & $0.93( \pm 0.29)^{\mathrm{ab}}$ & $0.62( \pm 0.22)^{\mathrm{a}}$ & $0.28( \pm 0.11)$ & $F(2,30)=88.11, n_{p}^{2}=.46, p<.01$ \\
$\mathrm{R}$ of trunk displacement $(\mathrm{cm})$ & $2.51( \pm 1.56)^{\mathrm{a}}$ & $2.71( \pm 2.00)^{\mathrm{a}}$ & $0.91( \pm 0.41)$ & $F(2,30)=12.87, n_{p}^{2}=.32, p<.01$ \\
SD of trunk displacement $(\mathrm{cm})$ & $0.62( \pm 0.48)^{\mathrm{a}}$ & $0.70( \pm 0.62)^{\mathrm{a}}$ & $0.21( \pm 0.11)$ & $F(2,30)=9.65, n_{p}^{2}=.28, p<.01$ \\
V of trunk displacement $\left(\mathrm{cm} . \mathrm{s}^{-1}\right)$ & $0.56( \pm 0.29)^{\mathrm{ab}}$ & $0.41( \pm 0.22)^{\mathrm{a}}$ & $0.22( \pm 0.09)$ & $F(2,30)=19.10, n_{p}^{2}=.36, p<.01$ \\
$S D C O P_{v}$ & $0.35( \pm 0.16)^{\mathrm{a}}$ & $0.37( \pm 0.20)^{\mathrm{a}}$ & $0.21( \pm 0.10)$ & $F(2,30)=9.41, n_{p}^{2}=.28, p<.01$ \\
$S D C O P_{c}$ & $0.15( \pm 0.13)^{\mathrm{a}}$ & $0.15( \pm 0.16)^{\mathrm{a}}$ & $0.06( \pm 0.03)$ & $F(2,30)=6.38, n_{p}^{2}=.23, p<.01$ \\
$\% S D C O P_{v} / C O P_{\text {net }}$ & $0.97( \pm 0.15)$ & $0.97( \pm 0.15)$ & $0.97( \pm 0.07)$ & $F(2,30)=0.02, p>.01$ \\
$\% S D C O P_{c} / C O P_{\text {net }}$ & $0.35( \pm 0.15)$ & $0.33( \pm 0.18)$ & $0.26( \pm 0.08)$ & $F(2,30)=2.59, p>.01$ \\
$C O P_{v}$ vs. $C O P_{\text {net }}$ & $0.93( \pm 0.05)$ & $0.93( \pm 0.07)$ & $0.96( \pm 0.04)$ & $F(2,30)=1.56, p>.01$ \\
$C O P_{c}$ vs. $C O P_{\text {net }}$ & $0.23( \pm 0.29)$ & $0.25( \pm 0.36)$ & $0.22( \pm 0.25)$ & $F(2,30)=0.04, p>.01$ \\
\hline
\end{tabular}

Note. $\mathrm{COP}=$ center of pressure; $\mathrm{R}=$ range; $\mathrm{SD}=$ standard deviation; $\mathrm{V}=$ mean velocity. $\mathrm{SD}$ amplitudes of the $\mathrm{COP}_{\mathrm{v}}\left(\mathrm{COP}_{\mathrm{vertical}}\right)$ and $\mathrm{COP}_{\mathrm{c}}$

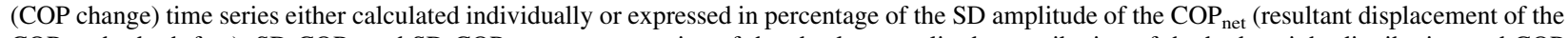
COP under both feet). SD $\mathrm{COP}_{\mathrm{v}}$ and $\mathrm{SD} \mathrm{COP}$ are representative of the absolute amplitude contribution of the bodyweight distribution and COP location mechanisms. $\% \mathrm{SD} \mathrm{COP} / \mathrm{COP}_{\text {net }}$ and $\% \mathrm{SD} \mathrm{COP} / \mathrm{COP}_{\text {net }}$ is representative of the relative amplitude contribution of these two mechanisms (see text). The table also shows cross-correlation coefficient in two analyses: $\mathrm{COP}_{v}$ versus $\mathrm{COP}_{\text {net }}$ and $\mathrm{COP}_{\mathrm{c}}$ versus $\mathrm{COP}_{\text {net }}$. These analyses are representative of the active contribution of both mechanisms (see text). The table shows averages $( \pm S D)$ in the $0.25 \mathrm{~Hz}$ and $0.125 \mathrm{~Hz}$ gaze shift conditions and in the stationary gaze condition.

${ }^{\mathrm{a} S}$ Significant difference between a gaze shift condition and the stationary gaze condition.

${ }^{\mathrm{b}}$ Significant difference between the two gaze shift conditions.

\section{Complementary Analyses}

Figures of the time series. A close look at the figures of the COP, back, trunk, and head time series showed that all these data were random-like in the stationary gaze conditions. For all the participants in the 0.125 and $0.25 \mathrm{~Hz}$ conditions, the neck time series mostly showed a square wave displacement of the marker (as the head-on-trunk displacement in
Figure 4). Only for half of the participants (8 of 16) in the 0.125 and $0.25 \mathrm{~Hz}$ conditions, the back time series showed a square wave displacement of the marker much less clearly pictured than the neck time series yet. The other half time series showed random-like displacement, that is with no square wave displacement-as the trunk-in-space displacement in Figure 4. The COP displacement was random-like in all trials, even in the 0.125 and $0.25 \mathrm{~Hz}$ conditions.

\begin{tabular}{|c|c|c|c|c|c|c|c|c|c|}
\hline & \multicolumn{3}{|c|}{$0.25 \mathrm{~Hz}$} & \multicolumn{3}{|c|}{$0.125 \mathrm{~Hz}$} & \multicolumn{3}{|c|}{ Stationary } \\
\hline & Trunk SD & Neck SD & Head SD & Trunk SD & Neck SD & Head SD & Trunk SD & Neck SD & Head SD \\
\hline $\begin{array}{l}C O P_{v} \text { versus } C O P_{\text {net }} \\
C O P_{c} \text { versus } C O P_{\text {net }}\end{array}$ & -.78 & -.68 & & -.86 & -.73 & & & & \\
\hline$S D C O P_{v}$ & .81 & .70 & & .79 & & & .97 & .90 & .89 \\
\hline $\begin{array}{l}S D C O P_{c} \\
\% S D C O P_{v} / C O P_{\text {net }}\end{array}$ & .76 & .68 & & .83 & .64 & & & & \\
\hline$\% S D C O P_{c} / C O P_{n e t}$ & .89 & .76 & & .88 & .75 & & & & \\
\hline
\end{tabular}

Note. The variables of angular body displacement (eyes, head, trunk) were also entered in the analyses but did not provide any significant relationship. Thus, they are not reported in the table. See Table 1 for the definition of these terms. All $r$ s are significant at $p<.01$. COP $=$ center of pressure; $\mathrm{SD}=$ standard deviation. 
Asymmetry. The analyses did not show any significant effect in the ML COP mean position, body marker mean position and load/unload weight repartition under each foot, $F_{s}(2,30)<1.21, p>.05$.

Controlling for confounding variables. We used the detrending normalization procedure recommended by O'Malley (1997) to remove the influence of age, height, and weight on the calculated time series. We performed again the ANOVAs on the normalized data. These analyses did not change any significant finding. They only slightly strengthened some results $\left(n_{p}^{2}\right.$ increased by .01-.03), mostly when controlling for weight. Hence, the participants' physical characteristics were not confounding variables.

\section{Discussion}

To perform the gaze shifts, the participants exhibited greater body marker displacements and greater COP displacements. Consistent with expectation, the two mechanisms contributed more to control ML body displacements in the gaze shift conditions than in the stationary gaze condition. However, the contribution of the bodyweight distribution mechanism tended to be weaker than expected, in relative terms, in gaze shift conditions. As we discuss, these results may show how ML postural control was adjusted to control ML stance while allowing body motions to perform the requested gaze shifts.

\section{The Tasks Were Well Performed}

In past published articles, visual performances in gaze shift conditions were always performed as requested (e.g., Anastasopoulos et al., 2009; Bonnet \& Despretz, 2012; Rey, Lê, Bertin, \& Kapoula, 2008; Sklavos et al., 2010; Stoffregen et al., 2007; Stoffregen et al., 2007). In the present study, the participants also performed the visual tasks, as requested in terms of amplitude, frequency, and timing. Thus, the participants organized their postural control to perform the task without losing their equilibrium. In the literature, some studies already analyzed postural coordination (eyes, head, lower back, feet movement) under large gaze shift conditions (Hollands et al., 2004; Anastopoulos et al., 2009; Sklavos et al., 2010). These studies with healthy, young adults showed that postural coordination was organized to facilitate gaze and head motions to reach the targets. Additionally, the higher the body part, the quicker the movement onset (Anastopoulos et al., 2009; Hollands et al., 2004). The eyes vs. head onset latency and the eyes versus foot amplitude displacement exhibited the greatest correlation coefficient (Hollands et al., 2004). We additionally showed that lower back and neck ML displacements were significantly greater in range and standard deviation in the active gaze shift conditions compared with the stationary gaze condition. As in Bonnet and Despretz (2012), the magnitude (range, SD, velocity) of COP displacement was significantly greater in the gaze shift conditions. This last finding was expected because the COMor body-displacement is known to be controlled by the COP displacement (Winter, 1995). The body displacement was greater in gaze shift conditions and the COP displacement necessarily needed to be greater. Thus, as in Bonnet and Despretz, the participants were actively stable in gaze shift conditions allowing greater magnitude of body displacements to perform the gaze shifts. These results were consistent with Hypothesis 1.

Several results showed that the $0.25 \mathrm{~Hz}$ condition was not clearly more challenging than the $0.125 \mathrm{~Hz}$ condition in healthy, young adults. Hence, Hypothesis 4 was not confirmed. Only one significant relationship between $\mathrm{SD} \mathrm{COP}_{v}$ and SD of the lower back displacement in the $0.25 \mathrm{~Hz}$ condition could not be found in the $0.125 \mathrm{~Hz}$ condition. The eye-in-orbit angular displacement was significantly lower in the $0.25 \mathrm{~Hz}$ than in the $0.125 \mathrm{~Hz}$ condition but the headon-trunk and trunk-in-space angular displacements were similar between these conditions. Hence, the heavy head and trunk segments coordinated similarly in both $0.25 \mathrm{~Hz}$ and $0.125 \mathrm{~Hz}$ conditions leaving only differences in no heavy body parts (the eyes). Moreover, the neck and lower back markers moved significantly quicker in the $0.25 \mathrm{~Hz}$ condition than in the $0.125 \mathrm{~Hz}$ condition (Table 1), most probably as a consequence of our method. Indeed, the figures of the data (cf. complementary analyses) showed twice more square wave like displacements of the markers in the $0.25 \mathrm{~Hz}$ condition than in the $0.125 \mathrm{~Hz}$ condition. These additional displacements necessarily increased the mean velocity of the markers. These displacements had no incidence on the magnitude ( $\mathrm{R}$ and SD) of COP, neck and lower back displacements, thus showing that the variability of ML body displacement was controlled similarly in both conditions. One should note that the postural control signal was more and more random-like from the top (eyes) to the bottom of the body (COP). In the rest of the present discussion, the results of both conditions will be combined. We now discuss how the two postural control mechanisms were adjusted to perform the visual tasks.

\section{Changes in the Contribution of the ML Bodyweight Distribution and COP Location Mechanisms}

The absolute amplitude contribution of both ML bodyweight distribution and COP location mechanisms was significantly greater in active gaze shift conditions than in the stationary gaze condition (Table 1). Hypothesis 2 was thus validated. We expected to find so to explain greater amplitude of COP displacement in gaze shift conditions. However, the relative amplitude contribution did not change significantly (Table 1). In other words, the increase in the amplitude contribution of both ML bodyweight distribution and COP location mechanisms were only proportional-not significantly greater or smaller-to the increase in COP displacement. The strength of postural control mechanisms was 
minimally adapted to the active task performed. By contrast in the literature of static studies, the relative amplitude contribution of both ML bodyweight distribution and COP location mechanisms was modified by changing the stance width. In Bonnet et al. (2013), it was found that the closer the feet, the lower the relative amplitude contributions of both mechanisms, probably because the less effective the force generated to control ML postural displacement (Henry, Fung, \& Horak, 2001; Winter et al., 1996).

In the present active study, we found approximately equal changes in the ML bodyweight distribution and COP location mechanisms. On the one hand, the significant change in amplitude contribution was slightly greater for the bodyweight distribution mechanism than for the COP location mechanism between the stationary and gaze shift conditions ( $n_{p}^{2}$ in Table 1$)$. On the other hand, the relationships between $\mathrm{COP}_{c}$ and body motions were stronger in gaze shift conditions while no such result was found for the bodyweight distribution mechanism. Surprisingly therefore, when ML postural control was actively challenged, the primary ML bodyweight distribution mechanism did not seem to change more than the secondary ML COP location mechanism to avoid losing equilibrium. One insight is that this prime mechanism may play another role than simply restraining ML body displacement, as we will suggest subsequently.

The active contribution of both ML bodyweight distribution and COP location mechanisms did not change between stationary and active conditions (no main effect in the ANOVA). Unexpectedly, significant negative relationships were found between $\mathrm{COP}_{v}$ versus $\mathrm{COP}_{\text {net }}$ and body marker displacements (lower back and neck) in the two gaze shift conditions (Table 2). Additionally, overall changes in $\mathrm{COP}_{v}$ and $\mathrm{COP}_{c}$ between conditions were weaker than changes in COP displacement, which were weaker than changes in body marker displacements (see $n_{p}^{2}$ in Table 1). These results were inconsistent with hypothesis 3. They were unexpected because changes in the controller were expected to be stronger than changes in the controlled variable to allow-by definition-adequate postural control (Maurer \& Peterka, 2005). These results may be key findings. Here in these active conditions, it may be that the controllers were slightly released (relative to a perfect control) to allow body motion to be performed in the visual tasks. We propose that if the contribution of the bodyweight distribution mechanism had been too high, individuals may have been too tensed to perform adequate body motions. In other words, once ML postural control was adjusted to objectively control the increase in body displacement in active conditions, slight release of the primary mechanism (pictured in the relative contribution of the bodyweight distribution mechanism) could be allowed to perform head and body motions. This a posterior interpretation does not seem to exist in the literature for one simple reason. Usually, researchers record and illustrate electromyographic (EMG) or other data in absolute terms but not in relative terms. Published studies have shown an increase in EMG activations once body motions have to be performed (e.g., Yiou, Caderby, \& Hussein, 2012) or when postural control has to be maintained under some kind of perturbations (e.g., Henry et al., 2001). However, these studies do not report differences between expected and measured EMG activations or other data. The possibility to analyze the absolute and relative amplitude contributions of the two mechanisms constitutes a real advantage of Winter et al.'s (1996; 1993) model to understand how these mechanisms work. In brief, our findings may show that the functioning of the ML postural control mechanisms is more complex in active than in static conditions.

\section{Perspectives and Conclusion}

A limitation of the present study is that the head, neck, and lower back rotations were not measured individually and thus not studied. Instead, we only get the head-in-space and trunk-in-space angular displacements with the SIMI Reality Motion System software. Also, the precision would have been better if we had used two markers at each level (head, shoulder, hip levels). However, our results were consistent with the condition requirements and thus validated our method. Indeed, the data showed that the participants moved their gaze (eyes+head-on-trunk+trunk-in-space angular displacements) $80.86^{\circ}$ and $82.37^{\circ}$ in the 0.25 and $0.125 \mathrm{~Hz}$ conditions, which is within the condition requirement $\left(80 \pm 2.5^{\circ}\right.$ on each side as the target covered $5^{\circ}$ of visual angle at their center).

In this study, we performed easier gaze shift conditions than in Bonnet and Despretz (2012) to obtain preliminary data for a future study with older adults (healthy and affected by Parkinson's disease). This future study will be relevant to better understand the nature of age- and diseaserelated deficiencies in ML postural control. Indeed, Termoz et al. (2008) already showed that postural control mechanisms are not clearly different between PD patients and their controls in static quiet stance while PD patients exhibit deficiencies in ML postural control in more challenging conditions (e.g., platform motion; cf. Adkin, Bloem, \& Allum, 2005; Horak, Dimitrova, \& Nutt, 2005).

\section{REFERENCES}

Adkin, A. L., Bloem, B. R., \& Allum, J. H. J. (2005). Trunk sway measurements during stance and gait tasks in Parkinson's disease. Gait and Posture, 22, 240-249.

Anastasopoulos, D., Ziavra, N., Hollands, M., \& Bronstein, A. (2009). Gaze displacement and inter-segmental coordination during large whole body voluntary rotations. Experimental Brain Research, 193, 323-336.

Bonnet, C. T., Cherraf, S., \& Do, M.-C. (2014a). Methodological requirement to analyze biomechanical postural control mechanisms with two platforms. Human Movement Science. doi:10.1016/j.humov.2014.03.007

Bonnet, C. T., Cherraf, S., Szaffarczyk, S., \& Rougier, P. (2014). The contribution of body weight distribution and center of 
pressure location in the control of mediolateral stance. Journal of Biomechanics, 47, 1603-1608.

Bonnet, C. T., \& Despretz, P. (2012). Large lateral head movements and postural control. Human Movement Science, 31, 1541-1551.

Bonnet, C. T., Mercier, M., \& Szaffarczyk, S. (2013). Impaired mediolateral postural control at the ankle in healthy, middleaged adults. Journal of Motor Behavior, 45, 333-342.

Day, B. L., Steiger, M. J., Thompson, P. D., \& Marsden, C. D. (1993). Effect of vision and stance width on human body motion when standing: Implications for afferent control of lateral sway. The Journal of Physiology, 469, 479-499.

Gatev, P., Thomas, S., Kepple, T., \& Hallett, M. (1999). Feedforward ankle strategy of balance during quiet stance in adults. Journal of Physiology, 514, 915-928.

Henry, S. H., Fung, J., \& Horak, F. B. (2001). Effect of stance width on multidirectional postural responses. Journal of Neurophysiology, 85, 559-570.

Hollands, M. A., Ziavra, N. V., \& Bronstein, A. M. (2004). A new paradigm to investigate the roles of head and eye movements in coordination of whole-body movements. Experimental Brain Research, 154, 261-266.

Horak, F. B., Dimitrova, D., \& Nutt, J. G. (2005). Direction-specific postural instability in subjects with Parkinson's disease. Experimental Neurology, 193, 504-521.

Lafond, D., Corriveau, H., \& Prince, F. (2004). Postural control mechanisms during quiet standing in patients with diabetic sensory neuropathy. Diabetes Care, 27, 173-178.

Maki, B. E., Holliday, P. J., \& Topper, A. K. A. (1994). Prospective study of postural balance and risk of falling in an ambulatory and independent elderly population. Journal of Gerontology, 49, M72-84.

Maurer, C., \& Peterka, R. J. (2005). A new interpretation of spontaneous sway measures based on a simple model of human postural control. Journal of Neurophysiology, 93, 189-200.

McIlroy, W. E., \& Maki, B. E. (1997). Preferred placement of the feet during quiet stance: Development of a standardized foot placement for balance testing. Clinical Biomechanics, 12, 66-70.

Mouzat, A., Dabonneville, M., \& Bertrand, P. (2004). The effect of feet position on orthostatic posture in a female sample group. Neuroscience Letters, 365, 79-82.

O'Malley, M. J. (1997). Normalization of temporal-distance parameters in pediatric gait. Journal of Biomechanics, 29, 619-625.

Rey, F., Lê, T.-T., Bertin, R., \& Kapoula, Z. (2008). Saccades horizontal or vertical at near or at far do not deteriorate postural control. Auris Nasus Larynx, 35, 185-191.
Riccio, G. E., \& Stoffregen, T. A. (1988). Affordances as constraints on the control of stance. Human Movement Science, 7 , 265-300.

Rogers, M. W., \& Mille, M.-L. (2003). Lateral Stability and Falls in Older People. Exercise and Sport Sciences Reviews, 31, 182-187.

Rougier, P. R. (2007). Relative contribution of the pressure variations under the feet and body weight distribution over both legs in the control of upright stance. Journal of Biomechanics, 40, 2477-2482.

Rougier, P. R. (2008) How spreading the forefeet apart influences upright standing control. Motor Control, 12, 362-374.

Sklavos, S., Anastasopoulos, D., \& Bronstein, A. (2010). Kinematic redundancy and variance of eye, head and trunk displacements during large horizontal gaze reorientations in standing humans. Experimental Brain Research, 202, 879-890.

Stoffregen, T. A., Bardy, B. G., Bonnet, C. T., Hove, P., \& Oullier, O. (2007). Postural sway and the frequency of horizontal eye movements. Motor Control, 11, 86-102.

Stoffregen, T. A., Bardy, B. G., Bonnet, C. T., \& Pagulayan, R. J. (2006). Postural stabilization of visually guided eye movements. Ecological Psychology, 18, 191-222.

Tarantola, J., Nardone, A., Tacclini, E., \& Schieppati, M. (1997). Human stance stability improves with the repetition of the task: Effect of foot position and visual condition. Neurosciences Letters, $228,75-78$

Termoz, N., Halliday, S. E., Winter, D. A., Frank, J. S., Patla, A. E., \& Prince, F. (2008). The control of upright stance in young, elderly and persons with Parkinson's disease. Gait and posture, 27, 463-470.

Winter, D. A. (1995). Human balance and postural control during standing and walking. Gait and Posture, 3, 193-214.

Winter, D. A., Prince, F., Frank, J. S., Powell, C., \& Zabjek, K. F. (1996). Unified theory regarding $\mathrm{A} / \mathrm{P}$ and $\mathrm{M} / \mathrm{L}$ balance in quiet stance. Journal of Neurophysiology, 75, 2334-2343.

Winter, D. A., Prince, F., Stergiou, P., \& Powell, C. (1993). Medial-lateral and anterior-posterior motor responses associated with center of pressure changes in quiet standing, Neuroscience Research Communications, 12, 141-148.

Yiou, E., Caderby, T., \& Hussein, T. (2012). Adaptability of anticipatory postural adjustments associated with voluntary movement. World Journal of Orthopedics, 3, 75-86.

Received October 2, 2013

Revised May 8, 2013

Accepted May 11, 2014 